\title{
Relationships Between Movements of the Lower Limb Joints and the Pelvis in Open and Closed Kinematic Chains During a Gait Cycle
}

\author{
by \\ Zdenek Svoboda ${ }^{1}$, Miroslav Janura ${ }^{1}$, Patrik Kutilek ${ }^{2}$, Eva Janurova ${ }^{3}$
}

Lots of athletic skills performed during practice or competition are initiated by the legs, where athletes either walk or run prior to executing specific skills. Kinematic chains are used to describe the relationships between body segments and joints during movement. The aim of this study was to determine the relationships between movements of lower limb segments and the pelvis in open and closed kinematic chains while walking. The experimental group consisted of 32 males (age $23.3 \pm 2.5$ years, body mass $78.1 \pm 8.7 \mathrm{~kg}$, body height $182 \pm 6 \mathrm{~cm}$ ). For $3 D$ analysis, an optoelectronic system Vicon MX (7 cameras, frequency $200 \mathrm{~Hz}$ ) was used. Positioning of the segments was determined by the PlugInGait Model. Each participant executed five trials at speeds ranging from 1.38 to $1.52 \mathrm{~m} \cdot \mathrm{s}^{-1}$. The relationships between angle variables of the lower limbs and the pelvis in selected gait cycle phases were evaluated using STATISTICA software (version 10.0) and the Spearman correlation. The highest numbers of moderate and large correlations were found at opposite toe off, heel rise and initial contact for the sagittal and transversal planes in comparison to the frontal plane. The closed kinematic chain had a stronger impact on determining the movement pattern. The instructions or interventions focusing on closed kinematic chain alternation are more effective for changes in a movement pattern. The preferred limb initiates kinematics in the direction of propulsion, while the non-preferred limb in internal and external rotation.

Key words: walking, coupling, intra-limb, coordination.

\section{Introduction}

Lots of athletic skills performed during practice or competition are initiated by the legs. Athletes either walk or run prior to executing specific skills (Panariello, 1991). The gait is a complex movement which includes both the open and closed kinematic chain. Therefore, the gait can be influenced by many factors such as age, the activity level, muscle activity (Bizovska et al., 2015; Cabell et al., 2013; Stastny et al., 2014, 2015), injury (Bunton et al., 1993) and disease (Krawczyk et al., 2012; Marchewka and Chwala 2007). In many studies, kinematic (Franz et al., 2009) and kinetic (Bovi et al., 2011; Cigali et al., 2004;
Jandačka et al., 2013b) variables during a gait cycle have been observed, but they were usually assessed independently in each joint.

From a mechanical point of view, while walking a human body can be considered a system of segments and joints forming various types of "chains" depending on the phase of a gait cycle. The first works on kinematic (kinetic) chains in this field were published in the 1870s. The authors of that time described kinematic chains as a system of overlapping rigid bodies linked by pivots. Upon external loading, force was transferred from one segment to an adjacent

1 - Faculty of Physical Culture, Palacky University Olomouc, Olomouc, Czech Republic.

2 - Faculty of Biomedical Engineering, Czech Technical University in Prague, Kladno, Czech Republic.

3 - Technical University of Ostrava, Czech Republic. 
segment, thereby enabling cumulative response of the whole chain (Reuleaux, 1875). In the 1950s, Arthur Steindler observed the possibilities of applying kinematic chains in the motor system (Steindler, 1977). One of the reasons for his interest was the finding that muscle activity in the lower limb with the foot fixed to the ground (a closed chain) was different when the foot was in free motion (an open chain), what also has implications in sports training.

In an open kinematic chain (OKC), the joint angle can be adjusted without incurring any changes in other joints. In a closed kinematic chain (CKC), however, both ends of the chain are fixed and any adjustment in the angle in one joint reciprocally results in altered angles in the other joint. A human gait, as other complex movements, typically consists of both the open and closed kinematic chains that frequently alternate during movement of the lower limbs. In the swing phase, the limbs adopt an open kinematic chain such that the distal segment movement velocity relates to the velocity of the proximal segment. Additionally, inertia acceleration in the distal segments is associated with deceleration of the proximal segments (McMullen and Uhl, 2000). During the stance phase (CKC), stability of the proximal segments depends on the stability afforded by the distal segments and foot instability influences the ability to stabilize the trunk along with proprioception from fixed parts of the body (Bunton et al., 1993).

In the scientific literature we can find many studies which observe interdependence between the movements of different limb segments during various movement tasks both in sport (Jandačka et al., 2013a; Sasaki et al., 2015) and clinical cases (Bunton et al., 1993; Khamis et al., 2015; Wright et al., 2008). However, when focused on walking, the authors mainly observed the relationships between parts of the foot and shank or knee (Dubbeldam et al., 2013; Pohl et al., 2007). Our study is more comprehensive and includes movements at the ankle, knee, hip and pelvis. With regard to sports training, a crucial question arises whether the CKC or OKC alternation would lead to parallel changes in the whole movement pattern.

The aims of the study were to describe the relationships between movements of the lower limb segments and the pelvis in open and closed kinematic chains while walking and to assess the effects of laterality on these relationships.

\section{Material and Methods}

\section{Participants}

The experimental group consisted of 32 males (age $23.3 \pm 2.5$ years, body mass $78.1 \pm 8.7 \mathrm{~kg}$, body height $182 \pm 6 \mathrm{~cm}$ ). Only participants with good health and no history of serious injury were included. The preferred limb was determined prior to measurements by asking which leg the participant would use to kick a ball.

\section{Experimental procedures and measures}

For 3D analysis, a Vicon MX (Vicon Motion Systems Ltd., Oxford, UK) optoelectronic system with seven infrared cameras (type T10, resolution 1 megapixel, frequency $200 \mathrm{~Hz}$ ) was used. The participants were asked to walk on an $8 \mathrm{~m}$ long walkway including two force plates (type: 9286AA, Kistler Instrumente AG, Winterthur, Switzerland). The positions of the lower limb segments and the pelvis were determined using the PlugInGait Model (16 markers) with consideration of marker placement error (Szczerbik and Kalinowska, 2011).

Each participant executed five trials at speeds ranging from 1.38 to $1.52 \mathrm{~m} \cdot \mathrm{s}-1$. This velocity range was determined during a preliminary study as the natural speed of the walk in young male subjects.

Angles at the ankle, knee, hip and pelvis in the sagittal, frontal and transversal planes were evaluated at specific gait cycle phases, as determined by force plates and the kinematic system as follows:

- initial contact (IC),

- opposite toe off (oTO),

- heel rise (HR),

- opposite initial contact (oIC),

- toe off (TO),

- maximal knee flexion (MKF),

- tibia in the vertical position (TV).

Ankle movement in the frontal plane (inversion/eversion) was not assessed since applying the conventional gait model in the absence of any enhancement in the set of foot markers is not considered reliable for this variable.

\section{Statistical analyses}

The kinematic data were processed using standard procedures by Vicon Nexus and Vicon 
Polygon software (Vicon Motion Systems Ltd., Oxford, UK).

The relationships between angle variables of the lower limbs and the pelvis (Table 1) in specific gait cycle phases were determined using STATISTICA software (version 10.0, StatSoft, Inc., Tulsa, OK, USA). The Kolmogorov-Smirnov test showed a non-normal data distribution, thus, the Spearman correlation coefficient was used. A correlation was considered large for values greater than 0.5 and moderate for values between 0.3 and 0.5 (Cohen, 1988). Positive correlations meant that movement in the joints occurred in the same direction (both for positive or negative). For example, a positive correlation between the ankle and the knee in the sagittal plane indicated that increased ankle dorsal flexion was associated with increased knee flexion. Conversely, a negative correlation illustrated a contrary joint movement; for example, in the transversal plane there was internal rotation at the ankle and external rotation at the knee.
For clarity, our study focused only on adjacent segments and the individual assessment of each plane.

\section{Results}

All large and moderate (greater than 0.3) correlations (Table 2) were statistically significant $(\mathrm{p}<0.05)$. The highest number of moderate and large correlations was found for the following phases of a closed kinematic chain: heel rise $(81 \%)$, opposite toe off and opposite initial contact $(63 \%)$. With regard to an open kinematic chain (maximal knee flexion $-38 \%$, tibia in the vertical position - 56\%) and borderline phases between closed and open kinematic chains (initial contact, toe off $-50 \%$ ), the number of large and moderate correlations was smaller.

The number of large and moderate correlations was slightly higher for the preferred limb (61\%) than for the non-preferred one (54\%). In regard to the movement planes, the number of correlations was higher in the sagittal (67\%) and transversal planes $(60 \%)$ compared to the frontal plane $(36 \%)$.

Table 1

\begin{tabular}{|c|c|c|c|c|c|c|}
\hline \multirow{2}{*}{$\begin{array}{c}\text { Joint } \\
\text { (segment) }\end{array}$} & \multicolumn{2}{|c|}{ Sagittal plane } & \multicolumn{2}{|c|}{ Frontal plane } & \multicolumn{2}{|c|}{ Transversal plane } \\
\hline & positive & negative & Positive & negative & positive & negative \\
\hline Ankle & $\begin{array}{l}\text { dorsal } \\
\text { flexion }\end{array}$ & $\begin{array}{l}\text { plantar } \\
\text { flexion }\end{array}$ & & & internal & external \\
\hline Knee & flexion & extension & Varus & valgus & internal & external \\
\hline Hip & flexion & extension & adduction & abduction & external & internal \\
\hline Pelvis & anteversion & retroversion & elevation & depression & internal & external \\
\hline
\end{tabular}




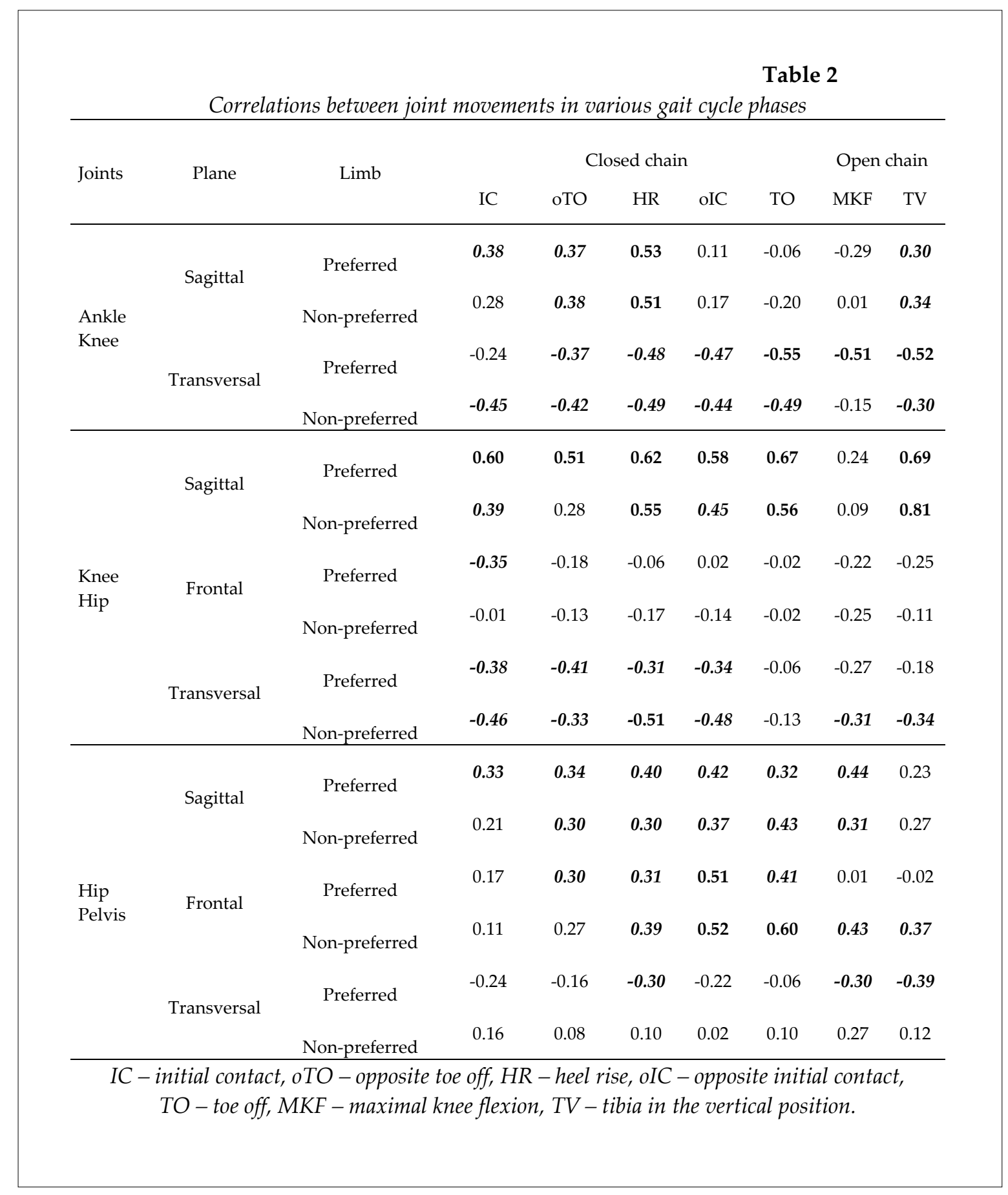

\section{Discussion}

The gait represents movement which combines both the OKC and CKC. This combination appears in most of sports movement patterns such as running or throwing, which have to be trained and improved in terms of movement stability. Kinematic chain segmental stability appears sequentially dependent, where the inferior segments are significantly correlated to the superior segments. Therefore, controlling lower limb segment stability may allow for increased stability at the more proximal segments and, ultimately, the trunk (Graham et al., 2011). Our results also show that segments coupling in relation to the OKC and CKC on the example of the gait present relationships that might be generalized for other basic movement patterns used in sports. Specifically, if the aim of the coach is to alternate the athletes movement pattern, it should be considered whether to alternate the 
CKC or OKC part of the movement. The same situation also appears when considering the benefits of eliciting changes in the preferred or non-preferred limb.

In the sagittal plane, our results showed significant correlations between adjacent segments during the entire stance phase (a closed chain) for the knee-hip and hip-pelvis pairs. For the ankle-knee pair, a significant correlation was found only for initial contact, opposite toe off and heel rise. These correlations are subject to changes at the knee joint, what is associated with braking of the body movement and reduced vertical displacement of the centre of mass (Rose and Gamble, 2006). During the swing phase (an open chain), significant correlations between the movements of the adjacent segments in the sagittal plane occur as well, however, values of the correlations and the number of the significant ones are smaller. Correlations were significant during MKF for the hip-pelvis pair and during TV for the ankle-knee and knee-hip pairs. A significant relationship between movement of the pelvis and the hip in the sagittal plane during a gait cycle was confirmed by Franz et al. (2009), who found a positive correlation between hip extension and the anterior pelvic tilt.

In the frontal plane, the highest number of significant correlations was found for the hippelvis pair. The highest values were found during a preswing (from oIC to TO) when the weight was transferred to the contralateral limb and the pelvis tilted towards the ipsilateral limb with associated hip abduction. For the pelvis to return to a neutral position during a midswing (between MKF and TV), simultaneous hip and knee flexion is necessary (Rose and Gamble, 2006; Whittle, 2007).

In the transversal plane, a significant correlation between the ankle-knee and knee-hip pairs was observed in almost all gait cycle phases. Since rotation in the knee joint is limited, we considered this fact as a correlation between the foot and the hip. Similar findings had been presented by Gaston et al. (2011) who revealed a correlation between transverse plane rotation at the foot and transverse plane rotation at the hip and the pelvis.
The highest number of moderate and large correlations was found for opposite toe off, heel rise and opposite initial contact. During all of these phases, the lower limb and the pelvis were in a closed kinematic chain. Zifchock et al. (2012) showed significantly greater coupling during the stance phase compared to the swing phase between the movements of the knee joint in the sagittal and transversal planes. Although coupling in this study means a movement of one joint in two planes, it suggests that different movements of the lower limbs correlate more in closed kinematic chains than in open ones.

The obtained results brought forward some differences in the correlations between the preferred and non-preferred limb. The number of moderate and large correlations was higher for the preferred limb. Between the knee and hip movements, the correlations were often larger in the preferred limb in the sagittal plane and larger in the non-preferred limb in the transversal plane. This suggests that functional differences between limbs depend on the contribution of each limb to performance of propulsion and control during able-bodied walking (Michalski et al., 2011; Sadeghi et al., 2000); the preferred limb appears to be more important for propulsion.

Some authors have presented correlations between non-adjacent segments and various planes (Svoboda et al., 2014). However, their studies mostly observed the influence of a foot position on more proximal segments.

\section{Conclusion}

Segments coupling during complex movements depend on both CKC and OKC movements, thus, alternation of each movement may change kinematics. Despite that, the CKC has a stronger influence on determining the movement pattern. Therefore, the instructions or interventions focusing on CKC alternation are more effective for movement pattern changes. Moreover, the preferred limb initiates kinematics in the direction of propulsion, while the nonpreferred limb in internal and external rotation.

\section{Acknowledgements}

The study was supported by a research grant from Czech Science Foundation (grant no. 15-13980S). 


\section{References}

Bizovska L, Svoboda Z, Janura M. The possibilities for dynamic stability assessment during gait: A review of the literature. Journal of Physical Education and Sport, 2015; 15: 490-497

Bovi G, Rabuffett M, Mazzoleni P, Ferrarin M. A multiple-task gait analysis approach: Kinematic, kinetic and EMG reference data for healthy young and adult subjects. Gait Posture, 2011; 33: 6-13

Bunton EE, Pitney WA, Cappaert TA, Kane AW. The Role of Limb Torque, Muscle Action and Proprioception During Closed Kinetic Chain Rehabilitation of The Lower Extremity. Journal of Athletic Training, 1993; 28: 10-20

Cabell L, Pienkowski D, Shapiro R, Janura M. Effect of age and activity level on lower extremity gait dynamics: an introductory study. J Strength Cond Res, 2013; 27: 1503-1510

Cigali BS, Ulucam E, Yilmaz A, Cakiroglu M. Comparison of asymmetries in ground reaction force patterns between normal human gait and football players. Biol Sport, 2004; 21: 241-248

Cohen J. Statistical power analysis for the behavioral sciences. 2nd ed. New York, NY: Academic Press; 1988

Dubbeldam R, Nester C, Nene AV, Hermens HJ, Buurke JH. Kinematic coupling relationships exist between non-adjacent segments of the foot and ankle of healthy subjects. Gait Posture, 2013; 37: 159-164

Franz JR, Paylo KW, Dicharry J, Riley PO, Kerrigan DC. Changes in the coordination of hip and pelvis kinematics with mode of locomotion. Gait Posture, 2009; 29: 494-498

Gaston MS, Rutz E, Dreher T, Brunner R. Transverse plane rotation of the foot and transverse hip and pelvic kinematics in diplegic cerebral palsy. Gait Posture, 2011; 34: 218-221

Graham RB, Costigan PA, Sadler EM, Stevenson JM. Local dynamic stability of the lifting kinematic chain. Gait Posture, 2011; 34: 561-563

Jandačka D, Estevan I, Janura M, Falco C. The impact of the initial stance position on lower limb joint kinetics in the taekwondo roundhouse kick. Acta Univ Palacki Olomuc Gymn, 2013; 43: 15-22

Jandačka D, Uchytil J, Svoboda Z, Elfmark M, Janura M. Test-retest reliability of the net joint power transferred by the lower limbs during walking in healthy men. Acta Univ Palacki Olomuc Gymn, 2013; 43: 7-15

Khamis S, Dar G, Peretz C, Yizhar Z. The Relationship Between Foot and Pelvic Alignment While Standing. J Hum Kinet, 2015; 46: 85-97

Krawczyk M, Syczewska M, Szczerbik E. Gait kinematics and clinical test changes in post-stroke patients during rehabilitation. Preliminary results of 12 patients of randomized clinical trial. Adv Rehabil, 2012; 26: $13-18$

Marchewka A, Chwala W. The analysis of gait in people with Down Syndrome-comparison with the norm in healthy people. Biol Sport, 2007; 24: 167-175

McMullen J, Uhl TL. A kinetic chain approach for shoulder rehabilitation. J Athl Train, 2000; 35: 329-337

Michalski R, Wit A, Gajewski J. Use of artificial neural networks for assessing parameters of gait symmetry. Acta Bioeng Biomech, 2011; 13: 66-70

Panariello RA. The closed kinetic chain in strength training. Strength Condit J, 1991; 13: 29-33

Pohl MB, Messenger N, Buckley JG. Forefoot, rearfoot and shank coupling: effect of variations in speed and mode of gait. Gait Posture, 2007; 25: 295-302

Reuleaux F. Theoretische Kinematik - Grundzüge einer Theorie des Maschinenwesens [Theoretical kinematics outline of a theory of machines]. Braunschweig: Von Fridrich Vieweg und Sohn; 1875

Rose J, Gamble JG. Human walking. 3rd ed. Philadelpha, PA: Lippincott Williams \& Wilkins; 2006

Sadeghi H, Allard P, Prince F, Labelle H. Symmetry and limb dominance in able-bodied gait: A review. Gait Posture, 2000; 12: 34-45

Sasaki S, Nagano Y, Kaneko S, Imamura S, Koabayshi T, Fukubayashi T. The Relationships Between the Center of Mass Position and the Trunk, Hip, and Knee Kinematics in the Sagittal Plane: A Pilot Study 
on Field-Based Video Analysis for Female Soccer Players. J Hum Kinet, 2015; 45: 71-80

Stastny P, Lehnert M, Zaatar A, Svoboda Z, Xaverova Z, Jelen K. Knee joint muscles neuromuscular activity during load-carrying walking. Neuroendocrinolo Lett, 2014; 35: 633-639

Stastny P, Tufano JJ, Lehnert M, Golas A, Zaatar A, Xaverova Z, Maszczyk A. Hip abductors and thigh muscles strength ratios and their relation to electromyography amplitude during split squat and walking lunge exercises. Acta Gymnica, 2015; 45: 51-59

Steindler A. Kinesiology of the human body under normal and pathological conditions. Springfield: Charles C Thomas Publisher; 1977

Svoboda Z, Honzikova L, Janura M, Vidal T, Martinaskova E. Kinematic gait analysis in children with valgus deformity of the hindfoot. Acta Bioeng Biomech, 2014; 16: 89-93

Szczerbik E, Kalinowska A. The influence of knee marker placement error on evaluation of gait kinematic parameters. Acta Bioeng Biomech, 2011; 13: 43-46

Whittle MW. Gait analysis: An introduction. 4th ed. Edinbourgh: Elsevier; 2007

Wright RW, Preston E, Fleming BC, Amendola A, Andrish JT, Bergfeld JA, Dunn WR, Kaeding C, Kuhn JE, Marx RG. A systematic review of anterior cruciate ligament reconstruction rehabilitation: part II: open versus closed kinetic chain exercises, neuromuscular electrical stimulation, accelerated rehabilitation, and miscellaneous topics. The journal of knee surgery, 2008; 21: 225-234

Zifchock BA, Pratt K, Brown A, Hillstrom H. Knee kinematic coupling in males and females: open and closed-chain tasks. J Appl Biomech, 2012; 28: 291-296

\section{Corresponding author:}

\section{Zdenek Svoboda, Ph.D.}

Department of Natural Sciences in Kinanthropology, Faculty of Physical Culture, Palacky University Olomouc

třída Míru 117, 77111 Olomouc, Czech Republic

Tel: +420 585636 414, Fax: +420 585412899

E-mail address: zdenek.svoboda@upol.cz 